\title{
1 Artificial intelligence assisted standard white light 2 endoscopy accurately characters early colorectal cancer: a \\ 3 multicenter diagnostic study
}

4 Sijun Meng ${ }^{1}, \mathrm{MD}, \mathrm{PhD}$, Yueping Zheng ${ }^{2}, \mathrm{MD}$, Ruizhang $\mathrm{Su}^{2}, \mathrm{MD}$, Wangyue Wang ${ }^{3,4}, \mathrm{MD}, \mathrm{Yu}_{\mathrm{Zhang}}{ }^{3}$,

$5 \mathrm{MD}, \mathrm{PhD}$, Hang $\mathrm{Xiao}^{5}, \mathrm{PhD}$, Zhaofang $\mathrm{Han}^{6,7}, \mathrm{PhD}$, Wen Zhang ${ }^{8}, \mathrm{PhD}$, Wenjuan $\mathrm{Qin}^{2}, \mathrm{PhD}, \mathrm{Chen}$

$6 \mathrm{Yang}^{5}, \mathrm{PhD}$, Lichong Yan ${ }^{9}, \mathrm{PhD}$, Haineng $\mathrm{Xu}^{10}, \mathrm{PhD}$, Yemei Bu ${ }^{5,11}$, MS , Yuhuan Zhong ${ }^{12}, \mathrm{PhD}, \mathrm{Yi}$

7 Zhang $^{13}$, MD, Yulong $\mathrm{He}^{1}, \mathrm{MD}$, PhD, Hesong Qiu ${ }^{6}$, MD, MBA, Wen $\mathrm{Xu}^{12}, \mathrm{PhD}$, Hong Chen ${ }^{1}$, MD,

$8{\text { Siqi } \mathrm{Wu}^{6,14}, \mathrm{PhD} \text {, Zhenghua Jiang }}^{15}$, BS , Yongxiu Zhang ${ }^{13}, \mathrm{MD}$, Chao Dong ${ }^{13}$, MD, Yongchao Hu ${ }^{13}$,

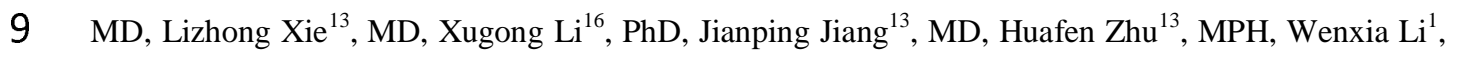

10 MSMT, Zhang Wen ${ }^{1}$, BSMT, Xiaofang Zheng ${ }^{13}$, BN, Yuanlong Sun ${ }^{4}$, MD, Xiaolu Zhou ${ }^{3}$, MD, Limin

$11 \mathrm{Ding}^{4}, \mathrm{MD}$, Changhua Zhang ${ }^{1 *}, \mathrm{MD}, \mathrm{PhD}$, Wensheng $\mathrm{Pan}^{3 *}, \mathrm{MD}$, Shuisheng Wu ${ }^{12}$ * $, \mathrm{MD}, \mathrm{Yiqun}^{\mathrm{H}} \mathrm{Hu}^{2}$

$12 *, \mathrm{MD}$

13

14 1. Center for Digestive Disease, the Seventh Affiliated Hospital of Sun Yat-sen University, Shenzhen,

15 Guangdong, Province, P. R. China, 518107.

16 2. Department of Gastroenterology, Zhongshan Hospital Xiamen University, 201 Hubin South Road,

17 Xiamen, Fujian Province, P. R. China, 361004. 
medRxiv preprint doi: https://doi.org/10.1101/2020.02.21.20025650; this version posted February 23, 2020. The copyright holder for this preprint (which was not certified by peer review) is the author/funder, who has granted medRxiv a license to display the preprint in perpetuity.

All rights reserved. No reuse allowed without permission.

18 3. Zhejiang provincial people's hospital, People's Hospital of Hangzhou Medical College, Hangzhou,

19 Zhejiang Province, P. R. China, 310014.

20 4. Tongxiang First People's Hospital, Tongxiang City, Zhejiang Province, P. R. China, 353000.

21 5. Jiying (XiaMen) Technology Co., LTD, Xiamen, Fujian Province, P. R. China, 361004.

22 6. Xiamen Cingene Science and technology co., LTD, Xiamen, Fujian Province, P. R. China, 361004.

23 7. State Key Laboratory of Marine Environmental Science, College of Ocean and Earth Sciences,

24 Xiamen University, Xiamen, P.R. China, 361102.

25 8. Friedman Brain Institute, Icahn School of Medicine at Mount Sinai, New York, NY, USA, 10029.

26 9. Department of Immunobiology, Yale University School of Medicine, New Haven, CT 06510, USA

27 10. University of Pennsylvania Perelman School of Medicine, Philadelphia, USA.

28 11. The School of Pharmacy in Fujian Medical University, Fujian Province, P. R. China.

29 12. College of Pharmacy, Fujian University of Traditional Chinese Medicine, Fuzhou, Fujian, P.R.

30 China, 350122.

31 13. Pucheng County Hospital of Traditional Chinese Medicine, Pucheng, Fujian Province, P. R. China,

32353400.

33 14. School of Life Sciences, Xiamen University, Xiamen, Fujian Province, P. R. China, 36110.

34 15. Department of Clinical Pharmacy, First Hospital of Nanping, Fujian Province, P. R. China, 314500

35 16. Department of Financial Engineering, Stevens Institute of Technology, Hoboken, NJ 07030, USA 


\section{$36{ }^{*}$ Correspondence to:}

37 Changhua Zhang: Center for Digestive Disease, the Seventh Affiliated Hospital of Sun Yat-sen

38 University, No. 628, Zhenyuan Rd, Guangming (New) Dist, Shenzhen, Guangdong Province, P. R.

39 China, 518107.E-mail: zhchangh@mail.sysu.edu.cn. Tel: +86-755-81206101.

40 Shuisheng Wu: College of Pharmacy, Fujian University of Traditional Chinese Medicine, No.1

41 Qiuyang Road, Shangjie University Town, Fuzhou, Fujian, P.R. China, 350122. E-mail:

42 Wushuishengwss@163.com. Tel: +86-591-22861135.

43 Wensheng Pan: Zhejiang provincial people's hospital, People's Hospital of Hangzhou Medical College,

44 No. 158 of Shangtang Road, XiaCheng District, Hangzhou, Zhejiang Province, P. R. China, 310014.

45 E-mail: wspan223@163.com. Tel: +86-571-87666666.

46 Yiqun Hu: Department of Gastroenterology, Zhongshan Hospital Xiamen University, 201 Hubin South

47 Road, Xiamen, Fujian Province, P. R. China, 361004. E-mail: hyq0826@xmu.edu.cn. Tel:

$48+86-592-2590151$. 


\section{ABSTRACT}

56 Colorectal cancer (CRC) is the third in incidence and mortality ${ }^{1}$ of cancer. Screening with colonoscopy

57 has been shown to reduce mortality by $40-60 \%^{2}$. Challenge for screening indistinguishable

58 precancerous and noninvasive lesion using conventional colonoscopy was still existing ${ }^{3}$. We propose to

59 establish a propagable artificial intelligence assisted high malignant potential early CRC

60 characterization system (ECRC-CAD). 4,390 endoscopic images of early CRC were used to establish

61 the model. The diagnostic accuracy of high malignant potential early CRC was 0.963 (95\% CI,

$620.941-0.978)$ in the internal validation set and 0.835 (95\% CI, 0.805-0.862) in external datasets. It

63 achieved better performance than the expert endoscopists. Spreading of ECRC-CAD to regions with

64 different medical levels can assist in CRC screening and prevention.

\section{Introduction}

66 Colorectal cancer (CRC) is one of the most common malignant tumors in the globe ${ }^{4}$. "Cancer Statistics,

672020 " indicates that CRC ranks the third in incidence and mortality ${ }^{1}$. Accurate identification and

68 treatment of early CRC have significantly reduced incidence and mortality ${ }^{5}$. However, early CRC is

69 generally asymptomatic, and coloscopy allows direct visual inspection of the intestinal tract and

70 same-session detection, biopsy, and subsequent removal of disease lesion. Several countries

71 recommend that adults over the age of 50 should regularly screen CRC by coloscopy ${ }^{6,7}$. While 
72 coloscopy is a kind of expert dependent examination, which can lead to missed diagnosis and

73 misdiagnosis due to various causes, such as the experience of endoscopist, the interference during

74 operation and inevitable interval of eye movement ${ }^{8}$. Computer assisted diagnose (CAD) system can

75 solve above-mentioned problems.

76 In recent years, some Artificial Intelligence (AI)-based CAD systems have come into the pre-clinical

77 application stage. However, the limitations of the research design (i.e. lack of external validation, not

78 solving the critical clinical problems) lead to the consequence that the existing assistances are not really

79 suitable for clinical practice directly ${ }^{9,10}$. In the field of gastrointestinal tumor, most studies focus on the

80 detection of polyps and adenomas ${ }^{11,12}$, the staging of advanced cancer ${ }^{13}$ and the optimizing of

81 endoscopic operation ${ }^{14,15}$. Effects of these CAD systems in decreasing mortality rate of CRC need to be

82 proven $^{16}$ by more clinical trials. Early gastrointestinal cancer diagnosis system that can directly

83 decrease the occurrence of CRC is limited ${ }^{17}$, because the quantity of images from a single medical

84 center is insufficient to support the establishment of the algorithm. Therefore, we aim to establish an

85 early CRC CAD and malignant potential characteristic system (herein referred to as ECRC-CAD)

86 based on standard white-light endoscopy (WLE), which can be clinically applied to different regions.

87 In this multi-center diagnostic clinical research, we establish an AI assisted early CRC diagnosis model

88 using deep learning, which is based on images of different quality endoscopy from hospitals in

89 different medical levels (i.e., rural, municipal, provincial) in China. Furthermore, ECRC-CAD can 
90 identify intraepithelial neoplasm (IN), which is one of the most typical malignant precancerous

91 conditions that can be differentiated by using image-enhanced endoscopy (IEE) and diagnosed by

92 histology test. The clinical applicability of this model has been verified in the internal and external

93 datasets collected retrospectively and anther internal dataset collected prospectively.

\section{Methods}

\section{Study design and participants}

96 This study was a multi-center clinical diagnose research, which was completed in five hospitals at

97 different medical levels across three provinces of China.

98 The workflow is shown in Figure 1. The first part is a case-control, retrospective research. All patients

99 who underwent coloscopy examination excepting for repeated examination in four hospitals from

100 January 2016 to July 2019 are enrolled, including Zhongshan Hospital of Xiamen University (XMZSH)

101 in Fujian, Zhejiang Provincial People's Hospital (ZJPH) in Zhejiang, the Seventh Affiliated Hospital of

102 Sun Yat-sen University (SYSUSH) in Guangdong, and Pucheng County Traditional Chinese Medicine

103 Hospital (PCTCRMH) in Fujian. Among them, SYSUSH started operation in July 2018. Patients who

104 were diagnosed with early CRC by histopathological examination were selected. Pathological

105 diagnosis was referred to the 4th WHO Classification of Tumors of Digestive System ${ }^{18}$. Early CRC in

106 this article referred to precancerous conditions, in accordance with the requirements of the international 
108 the database's including criteria (see in below) were included into case-control study, and were

109 randomly assigned to the training set, evaluation \& tuning set, and internal testing set in the ratio of

110 8:1:1. All images of intraepithelial neoplasm (referred as IN-ECRC) were included to case group, and

111 other early CRC without intraepithelial neoplasm (referred as NIN-ECRC) were randomly sample in

112 corresponding quantity included to control group. ECRC-CAD was constructed and optimized using

113 the first two data sets. To verify the veracity and clinical applicability of the model, we used validation

114 datasets including images from the above internal testing set and another external testing set, which are

115 consisted of images are according to the above criteria from Tongxiang Hospital (TXH) in Zhejiang.

116 The second part is a prospective research. The identification model officially completed the detection

117 and model optimization of the above samples in September 2019, and was connected to the above five

118 hospitals through the Internet. Then we prospectively collected the AI recognition results of all

119 endoscopic center images in October 2019 and the first half of November 2019 from the above five

120 hospitals, and extracted some endoscopic images of IN-ECRC and NIN-ECRC for senior doctors to

121 identify.

122 Each participating institute has got ethical approval by independent ethics Committee according to the

123 Helsinki declaration. The informed consent of retrospective cohort was exempted by the institutional

124 review boards, causes it's the secondary use of clinical data without any intervention. In the

125 prospective study, each patient was informed and signed up informed consent. 


\section{Endoscopy and early CRC's image database}

127 Endoscopic images of five hospitals were captured by EC590WM, EC-600WM, EC-L590Z (WFujinon

128 Corp, Saitama, Japan); V-70, PCF-H260AZI, CF-H260AI, CF-H290I, CF-HQ290I (Olympus Medical

129 Systems, Tokyo, Japan); EC34-i10M , EG2990Z I (PENTAX medical, Tokyo, Japan). Each image of

130 patients with early CRC was sequenced according to anatomical location, and screened by three

131 endoscopy physicians. Exclusion criteria include: 1. The endoscopic anatomical location was not

132 consistent with the pathological report, 2. Pictures were taken under IEE, 3. Pictures without any early

133 CRC lesion, 4. Pictures was not confirmed to the sampling standard ${ }^{19}$ summarized by our research team.

134 All images in the database present at least one lesion, and multiple images might be taken for the same

135 lesion, including differences of angle, distance, and extension of the intestinal wall. Images were saved

136 in a jpeg format.

\section{Image Labeling}

138 Two senior endoscopists with more than 10 years of clinical experience, four residents and two

139 assistants carried out image selection and labeling with blind method. The doctors were divided into

140 three groups randomly, and at least one senior doctor in each group. The deep learning marking tool

141 LabelMe ${ }^{20}$ of Anaconda was used to mark the outline of disease lesion manually. Two physicians in the

142 same group worked together. One was mainly responsible for drawing, and the other was mainly

143 responsible for checking. Only when two physicians from the same group reached a consensus, the 
144 labeling of the image could be finalized. After the first identification by ECRC-CAD, the misdiagnosed

145 images were double-checked so as to ensure the correctness and rationality of the results.

\section{Construction and optimize of algorithm}

147 The ECRC-CAD's algorithm was based on the concept of full convolution networks ${ }^{21}$ (FCN), which is

148 composed of classification module and post-processing module (Supplements p1-p2). The pattern

149 diagram was shown in Figure 2. Comparing with the classical CNN, this method can be adapted to

150 inputs with any size. In order to get an accurate boundary, we precisely outlined disease lesion of

151 images in establishing sets. The model contains one input and one output. The input of the model was

152 the image that contains the lesion. The output implemented a segmentation task that each pixel is

153 classified as background, intraepithelial neoplasm or other early CRC. Intraepithelial neoplasm was

154 abbreviated as "IN-ECRC", and the other early colorectal cancer neoplasm was abbreviated as

155 "NIN-ECRC". The labeling from histopathological examination was adopted as the gold standard in

156 training ECRC-CAD.

157 The implementation of AI-assisted precursor lesion identify model was fulfilled by using Python 3.7

158 (Python Software Foundation; www.python.org/).

\section{Algorithm testing and outcome measurement}

160 Three validation datasets were used to verify ECRC-CAD, which include internal datasets, external

161 datasets, and prospectively collected datasets. In the internal and external validate datasets, the 
162 performance of ECRC-CAD was compared with the pathological golden standard of the corresponding

163 tissues, and calculated statistic indicators on the individual images. When FCN detects early CRC from

164 the input data of the validation image, the model outputs the lesion name. When the output result of the

165 model is consistent with the pathological result, it is considered to be correct. In the prospective dataset,

166 the performance was compared with histopathological result and expert at the same time. We randomly

167 selected a subset of patients' images with histologically confirmed early CRC from the prospective

168 collected datasets. Two experienced endoscopists were asked to complete independently the same test

169 images together. These images' histology diagnosis is blinded to the two endoscopists. The diagnosis

170 results of ECRC-CAD and experts were taken the patient as the unit, which is according with clinical

171 requirements.

172 Sometimes, it's difficult to identify the whole images of disease lesion caused by the image quality and

173 angle. When the model can identify even one picture of the cancer, it is considered as correct.

\section{Statistical analysis}

175 In this study, descriptive analysis was used to describe the baseline characteristics of the enrolled

176 subjects. Continuous variables were expressed by median (interquartile interval), dichotomous

177 variables and grade variables that expressed by percentage. In order to evaluate the authenticity of

178 ECRC-CAD, the diagnostic evaluation method was adopted. The evaluating indexes included

179 Sensitivity, Specificity, Condolence, Positive predictive value (PPV), and Negative predictive value 
180 (NPV). According to the above indexes, receiver operating characteristic (ROC) of different subgroups

181 were drawn, and the area under the ROC curve (AUC) was calculated to evaluate the classification

182 effect of this model on different subjects. More details are in the supplements p3. All statistical

183 analyses in this study were based on the two-sided test 0.05 and performed through $\mathrm{R}$ software version

184 3.6.0 (R Development Core Team).

\section{Results}

\section{Patient and image dataset information}

187 More than 6.2 million patients have done lower gastrointestinal tract in XMZSH, ZJRMH, SYSUSH,

188 and PCRMH from January 2016 to August 2019. Almost 40\% percent without any disease lesion were

189 excluded. All participants diagnosed with IN-ECRC were enrolled in the model establishing sets, after

190 quality control, 1923 images were enrolled, participants with NIN-ECRC corresponding in number of

191 IN-ECRC were randomly extracted from other patients. The ratio of male to female is about 2.08:1.

192 Age ranges from 20 to 86 , and represent the right-skewed distribution (median age is 53.5). The

193 baseline information of the establishing cohort and external cohort were listed in Table 1. Finally, in

194 the stage of model establishment, 1923 images included in the case group, and 2467 images were

195 included in the control group. In the model optimization stage, 490 images were included. In the

196 verification stage, 189 images and 247 images were respectively collected in the above four hospitals

197 as internal datasets. External datasets, including 960 images from 134 patients, were used to contract 
198 and test ECRC-CAD. The prospectively collected datasets enrolled 672 patients, including 319

199 IN-ECRC and 353 NIN-ECRC patients.

200 Performance of the precancerous detection model

201 ECRC-CAD output results within 0.16 seconds and marks the boundary of disease lesion. The

202 diagnosis result of each image was listed in supplementary material (eTable 1). The diagnostic

203 accuracies of the internal validation dataset were 0.963 (95\% CI, 0.941 to 0.978 ). External testing set

204 only includes only IN-ECRC, which causes pathologists found that diagnostic criteria of NIN-ECRC in

205 TXH are not based on the 4th WHO Classification of Tumors of Digestive System. The sensitivity of

206 ECRC-CAD in THX is 0.835 (95\% IC, 0.805 to 0.862). The four indicates of the internal validation

207 dataset is higher than 0.93. Similarly, a high AUC value (0.939) was also observed in the internal

208 datasets (Figure 4). In the prospective study, the index of diagnosis accuracies was calculated in human

209 units, which is more suitable for clinical needs. High accuracy $(0.885,95 \%$ CI, 0.859 to 0.907$)$ was

210 observed. The sensitivity and PPV were more than 0.90 , and specificity and NPV were more than 0.85 .

211 The causes of misidentification are similarities, including incomplete exposure of disease lesions,

212 concomitant other diseases, and some normal structures. The PPV (0.609, 95\% IC, 0.454 to 0.745$)$ and

213 NPV $(0.426,95 \%$ IC, 0.303 to 0.559$)$ were significantly lower for the expert endoscopists when

214 compared with the AI-assisted model $(P<0.0001)$. Sensitivity and specificity between the endoscopic

215 experts and the ECRC-CAD were visible. 
216 This model has been successfully embedded in the above five hospitals, which is connected to the

217 endoscopically connected computers through the Internet. Doctors and clinicians can identify the

218 routine screenshots during the operation. The results of model recognition are displayed in the upper

219 right corner of the screen in real time. In order to benefit more people, ECE-CAD is now available at:

220 http://123.57.221.125/\#/home. Home page is on display in Supplementary material (eFigure 2).

221 Endoscopists can upload endoscopic images of suspected early CRC, and the system will give

222 judgment in real time. Furthermore, we provided a set of WLE images of early CRC through open

223 ports, which can help inexperienced endoscopist to familiar with it. The openness of this recognition

224 model can also help endoscopists in less developed areas or those with low seniority to learn the

225 recognition of early CRC (eFigure 3).

226 Both Asian and Weatern researchers consider that specific gross morphology, mucosal surface

227 structure and microvascular structure or multi-changes of neoplasm have corresponding molecular

228 characteristics $^{22}$. We analyzed the microstructure feature of WLE, IEE in the same lesion taken from

229 the same angle, and the association with features of H\&E stained pathological image (eFigure 4).

230 Combined with the published results (eTable 2), we proposed that the application of endoscopic images

231 to predict molecular characteristics through endoscopic or pathology images in real-time is the future

232 direction of "Image genomics"23.

\section{Discussion}


234 Overall, 1680 images of IN-ECRC and 2220 images of NIN-ECRC were used to establish and optimize

235 ECRC-CAD. In order to ensure the clinical application of the model in underdeveloped regions, these

236 images were collected from different medical level hospitals in China. Review published articles, it's

237 the largest, multi-source endoscopic image database used to establish AI aided diagnose system in

238 globe $^{24}$. Furthermore, three clinical validation studies were conducted on the immediate clinical

239 application value of ECRC-CAD, which showed high accuracy in the internal dataset (0.963). Liu et

$240 \mathrm{al}^{25}$ systematically reviewed the in-depth learning performance of diagnosis of disease from medical

241 imaging. Of the 82 eligible researches from 2012 to 2019 , only 25 have external validation. We

242 avoided the issues mentioned above in this meta-analysis. The displayed ECRC-CAD can be extended

243 to the external dataset from another rural hospital with a sensitivity of 0.835 . This verification cohort

244 could avoid highly accurate prediction which are caused by excessively spurious associations with the

245 known outcome. However, case-control would not be representative of the general screening

246 population $^{26}$, and the predictor may not be directly applied to clinical practice. In this study, we

247 validated ECRC-CAD in a prospective cohort, and ECRC-CAD and experts identified the disease's

248 nature in real-time, respectively. The result showed that ECRC-CAD maintained perfect accuracy and

249 specificity in prospective study, even better than expert endoscopist.

250 In clinical daily work, ECRC-CAD can assist endoscopist through increasing early CRC's detection

251 rate, decreasing missed diagnosis rate (Figure S5). Endoscopy and clinical practice of operator have a 
252 great influence on the detection and treatment of early cancer. A prospective study of $\mathrm{UK}^{27}$ shows that

253 the good performance of operating require skilled intubation operation and independent practice. While

254 endoscopist in developed region can get more information about microstructure under IEE, which can

255 increase diagnosis rate of early CRC by $10 \%-20 \%{ }^{28}$ comparing with WLE. However, early screening

256 of neoplasm is mostly carried out in community hospitals with relatively low medical level, and the

257 endoscopic equipment is relatively backward. Early CRC detection rate of ECRC-CAD has the same

258 performance with IEE. Using ECRC-CAD enable people in lower medical level regions to get similar

259 screening efficiency compared with subjects in developed regions.

260 Apart from assisting clinical decision, AI can make clinical path optimization possible ${ }^{23,29}$. The

261 introduction of colorectal endoscopic submucosal dissection (ESD) increased the complete resection

262 rate of early $\mathrm{CRC}^{30}$ with minimally invasive trauma and low local recurrence possibility. High-grade

263 IN without invasion or only superficial submucosal infiltration diagnosed by pathology examination is

264 an indicator for ESD. The interval time between first endoscopic biopsy and pathology diagnosis is

265 usually one week. At present, multi classification based on $\mathrm{IEE}^{31-33}$ has been widely used to predict the

266 risk of malignancy and invasiveness of CRC. Neoplasm confirm to indicators of malignant

267 aggressiveness subtype according to these classifications can be removed during first endoscopic

268 operation. It saved medical resources, and more important, absence of IEE or experts may increase

269 metastasis of implanted cancer cell in natural cavity ${ }^{34}$ due to incomplete resection. But there're some 
270 limitations: Kudo, Sano classification is based on single observation index of pit or vessel pattern,

271 NICE classification combining with gland and capillary pattern cannot differentiate IN from NIN, and

272 all classifications need expert practice and IEE. ECRC-CAD can identify IN under WLE within 0.16

273 second, which make up for the above deficiencies of present classification.

$274 \mathrm{WL} \mathrm{Bi}^{23}$ mentioned that characterization and monitoring are the future direction of $\mathrm{AI}$ in medical

275 image. ECRC-CAD have realized characterization of precancerous lesion, and it can steadily monitor

276 the progression of $\mathrm{CRC}$ without interference. However, with accumulation of medicine data

277 preternaturally over time, AI will add rich layers of intelligence through combining molecular and

278 pathological information with image-based findings. "Image genomics" ${ }^{123}$ is the key to realize it,

279 referring to the association of imaging characteristics with biological data, including specific molecular

280 markers that are mainly based on immunohistochemical, or sequencing technology that are developed

281 to direct personalized treatment planning. In lung cancers, researchers have established algorithm to

282 predicted EGFR genotype using computed tomography image (MRI). Can endoscopic images be used

283 in image genomics? We reviewed all researches of surface microstructure under endoscopy and

284 molecular characteristics associated with colorectal neoplasms. These results indicate that that the AI

285 have the potential of identify phenotypic and molecular specific pathological changes under WLE,

286 causes of AI can extract a large number of features that cannot be observed by the human naked eyes. 
287 The development of predicting multigroup tumor molecular markers through endoscopic images will

288 paved the way for the future "one-step" clinical workflow with AI interventions.

289 The insufficiencies in this study includes: 1. ECRC-CAD can't increase adenoma detection rate (ADR),

290 because it's based on static image taken by endoscopist. We've explored a CAD based on video, and

291 the clinical validation is processing. 2. The external validation set is not included with NIN-ECRC. We

292 are reassessing this part of cases by two expert histopathologists, to add a complete evaluation to this

293 model. 3. Sample of high-grade IN is limited for making differentiation of ECRC-CAD in low or

294 high-grade of IN. 4. We just focus on identifying IN-ECRC from NIN-ECRC, which can't distinguish

295 between sessile serrated lesions and other lesions. However, about $80 \%$ of sporadic CRC develop from

$296 \mathrm{IN}^{18}$, removal of which may decrease the incidence of colorectal cancer by $53 \%$. 5. The prospective

297 validation is not a randomized controlled trial (RCT). We're planning to proceed with subsequent RCT

298 studies in more centers. 6. We just put forward the endoscopic image that can be used to predict

299 molecular subtype, but ECRC-CAD cannot identify surface microstructure yet. Considering the clinical

300 applicability, the model was constructed using images taken by WLE in this study, which were not

301 suitable for micro-structure classification. In subsequent RCT studies, high-resolution WLE and IEE

302 images of early neoplasm will be prospectively collected to construct the classification model for 303 microstructure. 
304 This is an exploratory study on identification and diagnosis of early cancers that could be applied to

305 other organs of digestion system, such as the stomach and esophagus.

306

\section{Acknowledgement}

308 This research was supported by Major Science and Technology Project of Zhejiang Provincial

309 (2020C03030), Natural Science Foundation of Fujian Province (2019-CXB-31), Science and technology program of Guangdong Province (2016A020213002), and National Natural Science

311 Foundation of China (81773956). We would like to thank: (1) Yonghe Zhou, Kunping Hang for

312 coordinating the data in the primary hospitals; (2) Tonghai Dou, Ph.D, Chuncheng Cheng, ZI CHONG

313 KUO providing valuable advice; and Volunteer Joey Yau helps in data collation; (3) The Pucheng

314 county health administration has provided assistance to primary hospitals; (4)The families of all the

315 participants provided spiritual encouragement and material support, allowing researchers to continue

316 their experiments in the most difficult times.

\section{Contributors}

319 Yiqun $\mathrm{Hu}$ is general manager the of the project. Hesong Qiu is the project coordinator and participates

320 in the optimization of research design. Sijun Meng, Wangyue Wang, Yu Zhang, Zhaofang Han,

321 Wenjuan Qin, Yuhuan Zhong, Yi Zhang, Zhenghua Jiang, Yongxiu Zhang, Chao Dong, Yongchao Hu, 
medRxiv preprint doi: https://doi.org/10.1101/2020.02.21.20025650; this version posted February 23, 2020. The copyright holder for this preprint

(which was not certified by peer review) is the author/funder, who has granted medRxiv a license to display the preprint in perpetuity.

All rights reserved. No reuse allowed without permission.

322 Lizhong Xie, Jianping Jiang, Huafen Zhu, Wenxia Li, Zhang Wen, Xiaofang Zheng, Yuanlong Sun,

323 Xiaolu Zhou, Limin Ding have contributed in participants' enrollment, imaging collecting and quality

324 control. Yueping Zheng, Ruizhang Su, Siqi Wu, Yemei Bu, Xiaofang Zheng have classified and

325 integrated data. Hang Xiao and Chen Yang are responsible for algorithm establishing and optimizing.

326 Sijun Meng, Wen Zhang, Lichong Yan, Haineng Xu, and Xugong Li contribute to manuscript writing.

327 Ruizhang Su, Yemei Bu, Wenjuan Qin, Hong Chen, Wensheng Pan, Yiqun Hu were organized and

328 labeled photos. Yiqun Hu, Wensheng Pan, Hong Chen identified the IN-ECRC as expert endoscopist.

329 Shuisheng Wu, Wensheng Pan and Changhua Zhang's contributed equally in the project.

330 The corresponding author is Changhua Zhang, Shuisheng Wu, Wensheng Pan, Yiqun Hu. According to

331 the author ranking, Hesong Qiu and the previous author are first co-authors, Xugong Li and the

332 previous author are second co-authors, the others is the third co-authors.

333

\section{Ethical approval}

335 This study was approved by the medical ethic committee of each participants (IRB serial number:

336 XMZSYYKY_ER No.2019023).

\section{Declaration of interests}

339 We declare no competing interests. 


\section{Reference:}

342 1. Siegel, R.L., Miller, K.D. \& Jemal, A. Cancer statistics, 2020. CA: A Cancer

$343 \quad$ Journal for Clinicians 70, 7-30 (2020).

344 2. Brenner, H., Stock, C. \& Hoffmeister, M. Effect of screening sigmoidoscopy

345 and screening colonoscopy on colorectal cancer incidence and mortality:

346 systematic review and meta-analysis of randomised controlled trials and

347 observational studies. BMJ (Clinical research ed.) 348, g2467-g2467 (2014).

348 3. Su, M.-Y., et al. Comparative study of conventional colonoscopy,

349 chromoendoscopy, and narrow-band imaging systems in differential diagnosis

350 of neoplastic and nonneoplastic colonic polyps. The American journal of

351 gastroenterology 101, 2711-2716 (2006).

352 4. Bray, F., et al. Global cancer statistics 2018: GLOBOCAN estimates of

353 incidence and mortality worldwide for 36 cancers in 185 countries. $C A$ : $a$

354 cancer journal for clinicians 68, 394-424 (2018).

355 5. Lin, J.S., et al. Screening for Colorectal Cancer: Updated Evidence Report and

356 Systematic Review for the US Preventive Services Task Force. JAMA 315, 
358 6. Wolf AMD, F.E., Church TR, et al. . Colorectal cancer screening for

359 average-risk adults: 2018 guideline update from the American Cancer Society.

360 CA Cancer J Clin 68(4), 250-281 (2018).

361 7. Wender RC, B.O., Fedewa SA, Gansler T, Smith RA. . A blueprint for cancer

362 screening and early detection: Advancing screening's contribution to cancer

363 control. CA Cancer J Clin 69(1), 50-79 (2019).

364 8. Lee, J., et al. Risk factors of missed colorectal lesions after colonoscopy.

365 Medicine (Baltimore) 96, e7468-e7468 (2017).

366 9. Rajkomar A, D.J., Kohane I. Machine Learning in Medicine. N Engl J Med

$367 \quad 380(14), 1347-1358$ (2019).

368 10. AI diagnostics need attention. Nature 555(7696), 285 (2018).

369 11. Byrne, M.F., et al. Real-time differentiation of adenomatous and hyperplastic

370 diminutive colorectal polyps during analysis of unaltered videos of standard

371 colonoscopy using a deep learning model. Gut 68, 94-100 (2019).

372 12. Wang, P., et al. Real-time automatic detection system increases colonoscopic

373 polyp and adenoma detection rates: a prospective randomised controlled study.

$374 \quad$ Gut 68, 1813-1819 (2019). 
375 13. Zhu Y, W.Q., Xu MD, et al. Application of convolutional neural network in

376 the diagnosis of the invasion depth of gastric cancer based on conventional

377 endoscopy. Gastrointest Endosc 89(4), 806-815.e801. (2019).

378 14. Wu, L., et al. Randomised controlled trial of WISENSE, a real-time quality

379 improving system for monitoring blind spots during

380 esophagogastroduodenoscopy. Gut 68, 2161-2169 (2019).

381 15. Wu, L., et al. A deep neural network improves endoscopic detection of early

gastric cancer without blind spots. Endoscopy 51, 522-531 (2019).

383 16. Rees, C.J. \& Koo, S. Artificial intelligence - upping the game in

384 gastrointestinal endoscopy? Nature reviews. Gastroenterology \& hepatology

$385 \quad \mathbf{1 6}, 584-585(2019)$.

386 17. de Lange, T., Halvorsen, P. \& Riegler, M. Methodology to develop machine

387 learning algorithms to improve performance in gastrointestinal endoscopy.

$388 \quad$ World J Gastroenterol 24, 5057-5062 (2018).

389 18. Bosman, F.T., Carneiro, F., Hruban, R.H. \& Theise, N.D. WHO classification

$390 \quad$ of tumours of the digestive system, (World Health Organization, 2010).

391 19. Zheng, Y., et al. Setting Standards to Promote Artificial Intelligence in Colon 
393 20. Russell, B.C., Torralba, A., Murphy, K.P. \& Freeman, W.T. LabelMe: A

394 Database and Web-Based Tool for Image Annotation. International Journal of

$395 \quad$ Computer Vision 77, 157-173.

396 21. Shelhamer, E., Long, J. \& Darrell, T. Fully Convolutional Networks for

397 Semantic Segmentation. IEEE Transactions on Pattern Analysis and Machine

$398 \quad$ Intelligence 39, 640-651 (2017).

399 22. Suzuki, H., Yamamoto, E., Yamano, H.-O., Nakase, H. \& Sugai, T. Integrated

400 Analysis of the Endoscopic, Pathological and Molecular Characteristics of

401 Colorectal Tumorigenesis. Digestion 99, 33-38 (2019).

402 23. Bi, W.L., et al. Artificial intelligence in cancer imaging: Clinical challenges

403 and applications. CA: a cancer journal for clinicians 69, 127-157 (2019).

404 24. Ahmad, O.F., et al. Artificial intelligence and computer-aided diagnosis in

405 colonoscopy: current evidence and future directions. Lancet Gastroenterol

$406 \quad$ Hepatol 4, 71-80 (2019).

407 25. Liu, X., et al. A comparison of deep learning performance against health-care

408 professionals in detecting diseases from medical imaging: a systematic review

409 and meta-analysis. The Lancet Digital Health 1, e271-e297 (2019). 
410 26. Zhou, Q., Cao, Y.-H. \& Chen, Z.-H. Optimizing the study design of clinical

411 trials to identify the efficacy of artificial intelligence tools in clinical practices.

412 EClinicalMedicine 16, 10-11 (2019).

413 27. Siau, K., Hawkes, N.D., Dunckley, P. \& Valori, R.M. Impact of fellowship

414 training level on colonoscopy quality and efficiency metrics: a United

415 Kingdom perspective. Gastrointestinal endoscopy 89, 441-442 (2019).

416 28. Guo, T.-J., et al. Diagnostic performance of magnifying endoscopy with narrow-band imaging in differentiating neoplastic colorectal polyps from non-neoplastic colorectal polyps: a meta-analysis. Journal of gastroenterology 53, 701-711 (2018).

29. Rajkomar, A., Dean, J. \& Kohane, I. Machine Learning in Medicine. The New England journal of medicine 380, 1347-1358 (2019).

422 30. Sakamoto, T., et al. Endoscopic submucosal dissection for colorectal

423 neoplasms: a review. World journal of gastroenterology 20, 16153-16158 (2014).

425 31. Kudo, S., et al. Diagnosis of colorectal tumorous lesions by magnifying endoscopy. Gastrointest Endosc 44, 8-14 (1996). 
427 32. Sano, Y., et al. Magnifying observation of microvascular architecture of

428 colorectal lesions using a narrow $\square$ band imaging system. Digestive endoscopy

430 33. Hayashi, N., et al. Endoscopic prediction of deep submucosal invasive carcinoma: validation of the narrow-band imaging international colorectal endoscopic (NICE) classification. Gastrointestinal endoscopy 78, 625-632 (2013).

434 34. Lee, H.J., et al. Is endoscopic submucosal dissection safe for papillary

435 adenocarcinoma of the stomach? World journal of gastroenterology 21, 


\section{FIGURE LEGENDS}

449 Figure 1. Workflow of establishment and valuation of ECRC-CAD

450 The flow chart shows the construction and validation process and committed steps of ECRC-CAD.

451 Figure 2. Diagram of ECRC-CAD

452 Algorithm of ECRC-CAD is based on FCN8. Score of IN-ECRC+ score of NIN-ECRC=1, If score of

453 IN-ECRC > score of NIN-ECRC, we recognize it as IN-ECRC, if score of NIN-ECRC>score of

454 IN-ECRC, we recognize it as NIN-ECRC.

455 Figure 3. Demonstration of ECRC-CAD's extraction and characterization results

456 The left image of each pair is the original image under the endoscope, the right image of each pair is

457 the disease lesion extracted by ECRC-CAD. Predict result was listed under each pair of images. The

458 "Prediction" result represents the type of extracted lesion identified by ECRC-CAD, the "confidence"

represents the score that were calculated by ECRC-CAD.

460 Figure 4. Receiver operating characteristic curves of ECRC-CAD

461 ECRC-CAD performance on the internal validate sets (436 images). AUC indicates area under the 
medRxiv preprint doi: https://doi.org/10.1101/2020.02.21.20025650; this version posted February 23, 2020. The copyright holder for this preprint (which was not certified by peer review) is the author/funder, who has granted medRxiv a license to display the preprint in perpetuity.

All rights reserved. No reuse allowed without permission.

\section{3}

464 
medRxiv preprint doi: https://doi.org/10.1101/2020.02.21.20025650; this version posted February 23, 2020. The copyright holder for this preprint (which was not certified by peer review) is the author/funder, who has granted medRxiv a license to display the preprint in perpetuity.

All rights reserved. No reuse allowed without permission.

\section{FIGURES}

\section{Figure 1. Workflow of establishment and valuation of ECRC-CAD}

467

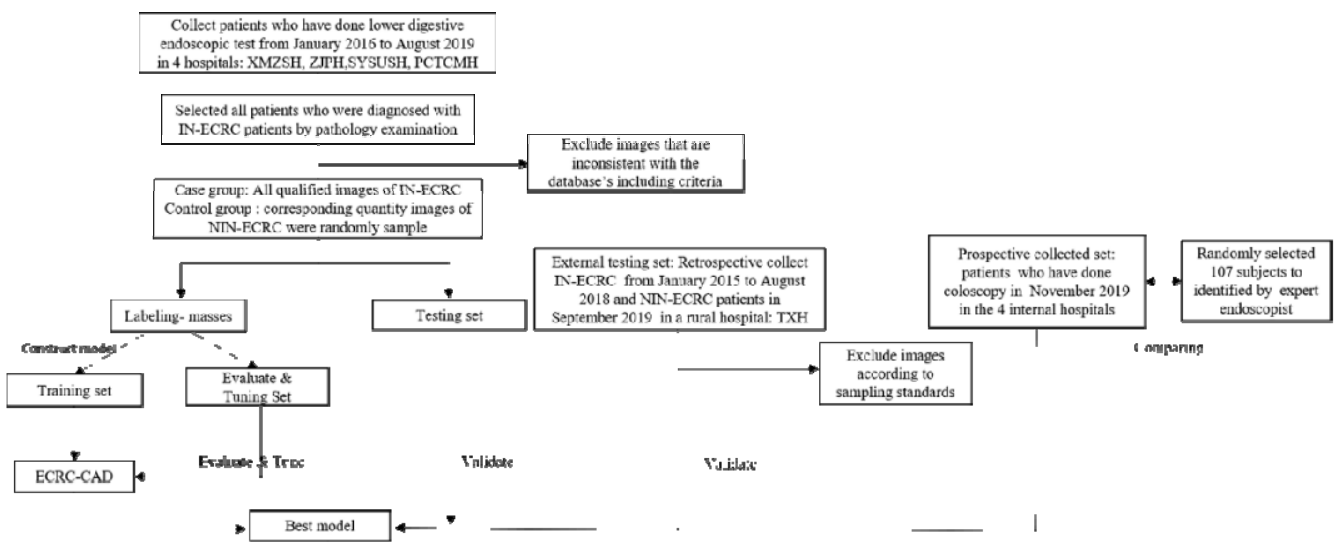

468

469 Figure 2. Diagram of ECRC-CAD
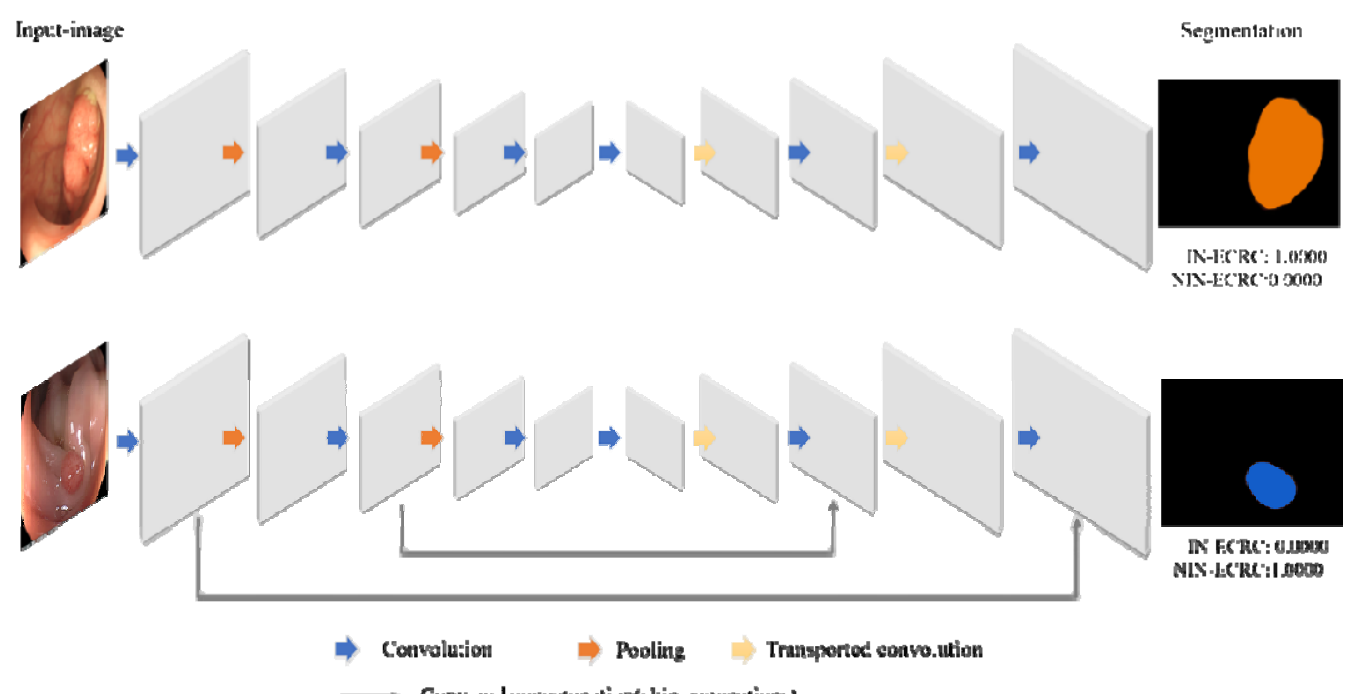
medRxiv preprint doi: https://doi.org/10.1101/2020.02.21.20025650; this version posted February 23, 2020. The copyright holder for this preprint (which was not certified by peer review) is the author/funder, who has granted medRxiv a license to display the preprint in perpetuity.

All rights reserved. No reuse allowed without permission.

474 Figure 3. Demonstration of ECRC-CAD's extraction and characterization

\section{5 results}

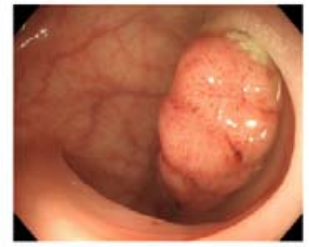

Prediction:IN-ECRC; Confidence: 1.000

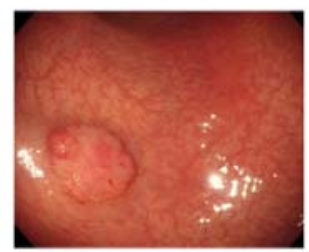

Prediction:IN-ECRC ; Confidence: 1000

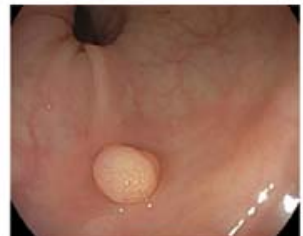

Prediction:NIN-ECRC; Confidence: 0.615

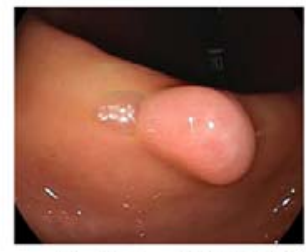

Prediction:NIN-ECRC ; Confidence: 0.803
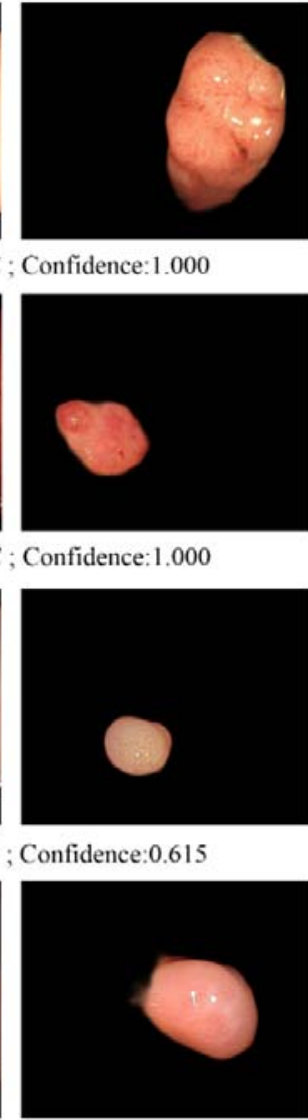

476
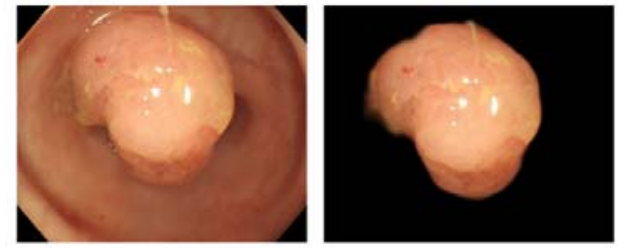

Prediction:IN-ECRC ; Confidence:0.910
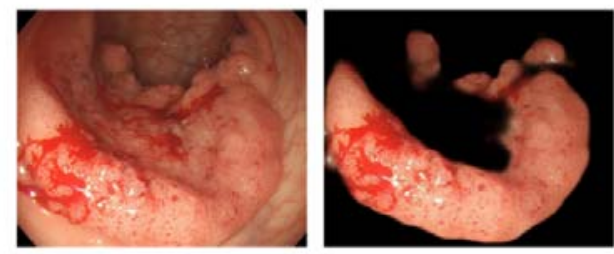

Prediction:IN-ECRC ; Confidence:0.910
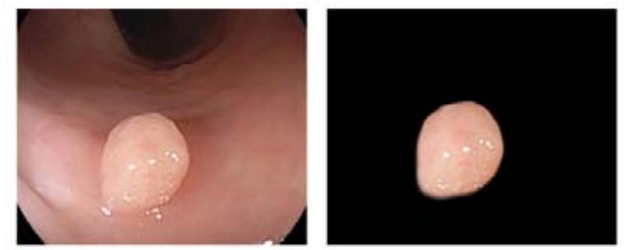

Prediction:NIN-ECRC ; Confidence:0.956
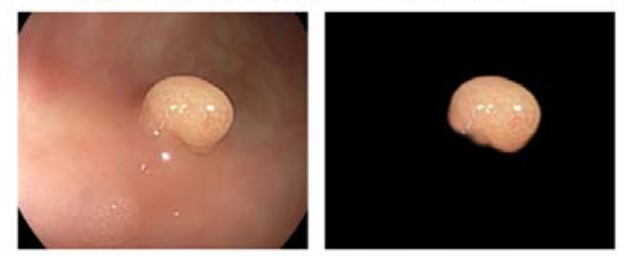

Prediction:NIN-ECRC ; Confidence: 1.000

477 Figure 4. Receiver operating characteristic curves of ECRC-CAD 
medRxiv preprint doi: https://doi.org/10.1101/2020.02.21.20025650; this version posted February 23, 2020. The copyright holder for this preprint (which was not certified by peer review) is the author/funder, who has granted medRxiv a license to display the preprint in perpetuity.

All rights reserved. No reuse allowed without permission.

\section{8}

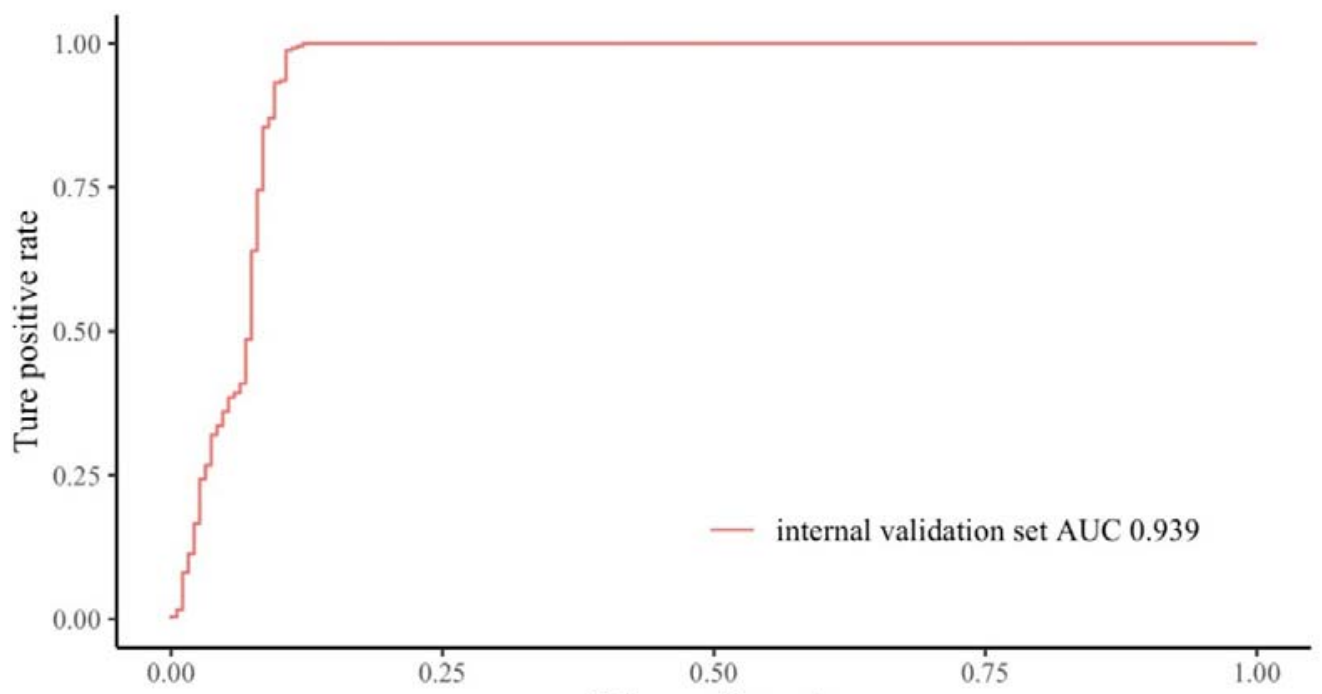

False positive rate 
Table 1. Baseline information

\begin{tabular}{|c|c|c|c|c|c|c|c|}
\hline & \multicolumn{3}{|c|}{ Establish cohort } & \multicolumn{2}{|c|}{ Validate cohort } & \multirow{2}{*}{$\begin{array}{l}\text { Expert } \\
\text { endoscopists } \\
\text { (unit: person) }\end{array}$} \\
\hline & & $\begin{array}{l}\text { Establishing set } \\
\text { (unit: image) }\end{array}$ & $\begin{array}{l}\text { Evaluate \& Tuning } \\
\text { set (unit: image) }\end{array}$ & $\begin{array}{l}\text { Internal testing set } \\
\text { (unit: image) }\end{array}$ & $\begin{array}{l}\text { External testing set } \\
\text { (unit: image) }\end{array}$ & $\begin{array}{l}\text { Prospective testing set } \\
\text { (unit: person) }\end{array}$ & \\
\hline \multicolumn{2}{|c|}{ Sum of subjects } & 3464 & 490 & 436 & 674 & 454 & 107 \\
\hline \multirow[t]{2}{*}{ Stage } & IN-ECRC & 1491 & 243 & 189 & 674 & 98 & 63 \\
\hline & NIN-ECRC & 1973 & 247 & 247 & $\mathrm{NaN}$ & 353 & 44 \\
\hline
\end{tabular}

480 
489 Table 2. Performance of ECRC-CAD in different validation dataset and expert endoscopist in distinguishing IN-ECRC and NIN-ECRC

\begin{tabular}{|l|l|l|l|l|l|}
\hline & Internal testing set & External testing set & Prospective testing set & Expert endoscopist & $\begin{array}{l}\text { ECRC-CAD vs } \\
\text { Endoscopist* }\end{array}$ \\
\hline Accuracy (95\% CI) & $0.963(0.941-0.978)$ & $0.835(0.805-0.862)$ & $0.885(0.859-0.907)$ & $0.505(0.411-0.598)$ & $P<0.0001$ \\
\hline Sensitivity (95\% CI) & $0.937(0.889-0.965)$ & $0.835(0.805-0.862)$ & $0.915(0.878-0.942)$ & $0.444(0.321-0.575)$ & $P<0.0001$ \\
\hline Specificity (95\% CI) & $0.984(0.956-0.995)$ & NaN & $0.858(0.817-0.892)$ & $0.591(0.433-0.733)$ & $P<0.0001$ \\
\hline PPV (95\% CI) & $0.978(0.941-0.993)$ & NaN & $0.854(0.811-0.887)$ & $0.609(0.454-0.745)$ & $P<0.0001$ \\
\hline NPV (95\% CI) & $0.953(0.917-0.974)$ & NaN & $0.918(0.882-0.944)$ & $0.426(0.303-0.559)$ & $P<0.0001$ \\
\hline
\end{tabular}

*McNemar's test 